\title{
Carpal Tunnel Syndrome Assessment With Ultrasonography: A Comparison Between Non-diabetic and Diabetic Patients
}

\author{
Chung Ho Lee, MD, Hanboram Choi, MD, Joon Shilk Yoon, MD, PhD, Seok Kang, MD \\ Department of Physical Medicine and Rehabilitation, Korea University Guro Hospital, Seoul, Korea
}

\begin{abstract}
Objective To investigate the diagnostic value of cross-sectional area (CSA) and wrist to forearm ratio (WFR) in patients with electro-diagnosed carpal tunnel syndrome (CTS) with or without diabetes mellitus (DM).

Methods We retrospectively studied 256 CTS wrists and 77 healthy wrists in a single center between January 1, 2008 and January 1, 2013. The CSA and WFR were calculated for each wrist. Patients were classified into four groups according to the presence of DM and CTS: group 1, non-DM and non-CTS patients; group 2, non-DM and CTS patients; group 3, DM and non-CTS patients; and group 4, DM and CTS patients. To determine the optimal cut-off value, receiver operating characteristic (ROC) curve analysis was performed.

Results The CSA and WFR were significantly different among the groups $(\mathrm{p}<0.001)$. The ROC curve analysis of non-DM patients revealed CSA $\geq 10.0 \mathrm{~mm}^{2}$ and WFR $\geq 1.52$ as the most powerful diagnostic values of CTS. The ROC curve analysis revealed CSA $\geq 12.5 \mathrm{~mm}^{2}$ and WFR $\geq 1.87$ as the most powerful diagnostic values of CTS.

Conclusion Ultrasonographic assessment for the diagnosis of CTS requires a particular cut-off value for diabetic patients. Based on the ROC analysis results, improved accurate diagnosis is possible if WFR can be applied regardless of presence or absence of DM.
\end{abstract}

Keywords Carpal tunnel syndrome, Ultrasonography, Electrodiagnosis, Diabetes mellitus

\section{INTRODUCTION}

Carpal tunnel syndrome (CTS) is the most common entrapment disorder, characterized by combinations of specific clinical presentations [1]. Currently, diagnosis is made based on characteristics symptoms and confirmed by nerve conduction studies and electromyography. In clinical practice, the electro-diagnostic (EDX) test is the most common and reliable evaluation method though it is uncomfortable and does not provide anatomic information about the median nerve and surrounding tissues. More recently, ultrasonography has been used to diag-

Received June 19, 2017; Accepted July 19, 2017

Corresponding author: Seok Kang

Department of Physical Medicine and Rehabilitation, Korea University Guro Hospital, 148 Gurodong-ro, Guro-gu, Seoul 08308, Korea. Tel: +82-2-26261500, Fax: +82-2-2626-1513, E-mail: caprock8@daum.net

ORCID: Chung Ho Lee (https://orcid.org/0000-0001-5140-2841); Hanboram Choi (https://orcid.org/0000-0001-9779-3921); Joon Shik Yoon (https:// orcid.org/0000-0002-8985-279X); Seok Kang (https://orcid.org/0000-0001-5614-4175).

() This is an open-access article distributed under the terms of the Creative Commons Attribution Non-Commercial License (http://creativecommons.org/ licenses/by-nc/4.0) which permits unrestricted noncommercial use, distribution, and reproduction in any medium, provided the original work is properly cited. Copyright $\odot 2018$ by Korean Academy of Rehabilitation Medicine 
nose CTS because it is non-invasive, easily accessible, and provides spatial information $[2,3]$.

Diabetes mellitus (DM) is reported as a significant independent risk factor for CTS [4]. Some studies reported peripheral nerve changes in DM patients and described enlarged median nerve cross-sectional area (CSA) regardless of the presence of peripheral polyneuropathy [5]. However, other reports showed no significant difference in CSA in the wrist in DM patients with axonal (including diabetic) polyneuropathy compared to that of healthy volunteers $[6,7]$. At this point, whether the median nerve is altered in patients with DM remains unclear. In addition, the specific criteria for the application of ultrasonography in DM patients have not been well established.

In this study, we aimed to assess the ultrasonographic changes in the median nerve between non-DM and DM patients with CTS. The diagnostic value of the ultrasonographic parameters in the discrimination between nonDM and DM cases was also investigated.

\section{MATERIALS AND METHODS}

\section{Subjects}

This study was a retrospective observational study of patients who underwent electrodiagnostic and ultrasonographic tests for the diagnosis of CTS. We carried out a retrospective analysis of 256 wrists with EDX evidence of CTS and 97 wrists without CTS in a single center between January 1, 2008 and January 1, 2013. DM was diagnosed according to the World Health Organization criteria [8]. Exclusion criteria for the study were as follows. (1) Patients with a clinical and electrophysiologic diagnosis of diabetic polyneuropathy. Clinical diagnosis was diagnosed based on the presence of one symptom (e.g., numbness, tingling, weakness, foot pain, or ataxia) or one sign (e.g., abnormal knee or ankle reflex). Diabetic polyneuropathy was electrophysiologically diagnosed by modified versions of the criteria suggested in the Diabetes Control and Complication Trial (Table 1). And (2) Patients who underwent prior surgery for CTS, patients with cervical radiculopathy, and patients with any underlying metabolic disorders such as alcoholism, genetic disorders, gout, rheumatic arthritis or abnormal thyroid function related to peripheral neuropathy.

Patients were classified into four groups according to the presence of DM and CTS: group 1, non-DM and non-
Table 1. Electrophysiological criteria for diabetic polyneuropathy

\begin{tabular}{ll}
\hline \multicolumn{1}{c}{ Parameter } & \multicolumn{1}{c}{ Value } \\
\hline Sural SNAP amplitude & $\leq 5 \mu \mathrm{V}$ \\
Median SNAP amplitude & $\leq 10 \mu \mathrm{V}$ \\
Peroneal CMAP amplitude & $<1 \mathrm{mV}$ \\
Peroneal NCV & Distal latency $\geq 6 \mathrm{~ms}$ or \\
& $\mathrm{NCV}<40 \mathrm{~m} / \mathrm{s}$ \\
Peroneal F-wave latency & Absent or $>55 \mathrm{~ms}$ \\
H-reflex & Absent \\
Needle EMG & Fibrillations in lower \\
& extremity muscles (TA, \\
& GCM, etc.) \\
\hline
\end{tabular}

Probable: 1 (sural SNAP $\leq 5 \mu \mathrm{V}$ ) plus at least 2 of the above indicators.

Definite: probable plus at least 2 of the other indicators; 1 (sural SNAP $\leq 5 \mu \mathrm{V}$ ) plus at least 4 of the indicators.

Modified criteria of the Department of Physical Medicine and Rehabilitation, Korea University College of Medicine presented by "The Diabetes Control and Complications Trial Research Group" in 1995.

SNAP, sensory nerve action potential; CMAP, compound motor nerve action potential; NCV, nerve conduction velocity; EMG, electromyography; TA, tibialis anterior; GCM, medial gastrocnemius.

CTS patients; group 2, non-DM and CTS diagnosed patients; group 3, DM and non-CTS patients; and group 4, DM and CTS diagnosed patients.

\section{Electro-diagnostic testing}

The nerve conduction study was conducted using Nicolet Viking IV (Nicolet Biomedical, Madison, WI, USA). Skin temperature was maintained above $32^{\circ} \mathrm{C}$. Standard tests included median sensory nerve conduction velocity within the 3rd digit/wrist segments and median distal motor latency from the wrist to the thenar eminence. When the standard tests were normal, further segmental tests were performed over a short distance of $7 \mathrm{~cm}$ or comparative median/ulnar studies. A median sensory nerve action potential was employed to record the initial latency and baseline to peak amplitude from the 3rd finger (active site), with retrograde stimulation being conducted at $14 \mathrm{~cm}$ proximal area from the recording site (wrist) and at $7 \mathrm{~cm}$ proximal area from the recording site (palm). The EDX criteria of CTS were determined as (1) median nerve distal sensory latency, upper limit of nor- 
mal $3.7 \mathrm{~ms}$; (2) difference between the median and ulnar nerve distal sensory latencies, upper limit of normal 0.4 ms; (3) distal motor latency over the thenar eminence, upper limit of normal $4.2 \mathrm{~ms}$; (4) median motor nerve conduction velocity, lower limit of normal $49 \mathrm{~m} / \mathrm{s}$; (5) median sensory nerve conduction velocity, lower limit of normal $49 \mathrm{~m} / \mathrm{s}$; and (6) a ratio of proximal and distal onset latency $>2.0$.

\section{Ultrasonography assessment}

After the EDX, the median nerve on the wrist was examined with ultrasound performed by a skilled physiatrist with neuromuscular ultrasound experience. A physician, blinded to the EDX findings and clinical parameters, evaluated the ultrasonographic results. Imaging was performed using the HD15 ultrasonography system (Philips, Bothell, WA, USA) and a 7-12 MHz linear array transducer. The ultrasonography settings were optimized for nerve imaging, such as frequency, depth, and focal zone. The probe was maintained at a perpendicular angle during analysis to prevent anisotropy and median nerve deformation, and additional weight was not permitted when the pressure was applied to the skin surface with the probe. The CSA of the median nerve was measured by direct tracing with electronic calipers around the nerve circumference, excluding the hyperechoic epineuria rim. CSA was measured at two locations. The two locations of the median nerve ultrasonographic parameters included the maximal swelling point of the median nerve around the wrist and $12 \mathrm{~cm}$ proximal to the maximal swelling point (Fig. 1). Following measurement, the CSA and the wrist to forearm ratio (WFR, defined as the ratio of the median nerve CSA at the maximal swelling site to its CSA at the $12 \mathrm{~cm}$ proximal site) were calculated.

\section{Statistical analysis}

Statistical analysis was performed using the SPSS statistical software version 22 for Windows (IBM, Armonk, NY, USA). All parameters were presented as the mean \pm standard deviation and compared among the groups. One-way ANOVA analysis was used to evaluate the differences in baseline characteristics among the groups. We used the analysis of covariance (ANCOVA) adjusting for baseline outcome values, for the comparison of the CSA and WFR. For post hoc testing, we used the Bonferroni correction. Receiver operating characteristics (ROC) curve analysis was used to determine the predictive CSA value for CTS. A p-value less than 0.05 was considered statistically significant.

\section{RESULTS}

\section{Baseline characteristics}

The baseline characteristics of the study subjects are shown in Table 2. Age, sex, height, and body mass index (BMI) were significantly different among the groups $(\mathrm{p}<0.0001)$, but there was no significant difference in weight among the groups.

\section{Comparison of ultrasonographic measurements among} the groups

Ultrasonographic measurements among the groups are shown in Table 3. The median nerve CSA at the maximal swelling point and the WFR were significantly different among the four groups $(\mathrm{p}<0.001)$. The CSA values did not show statistically significant differences between groups 2 and 3; however, when the WFR was applied, statistically significant differences were observed between groups 2 and $3(\mathrm{p}<0.001)$.
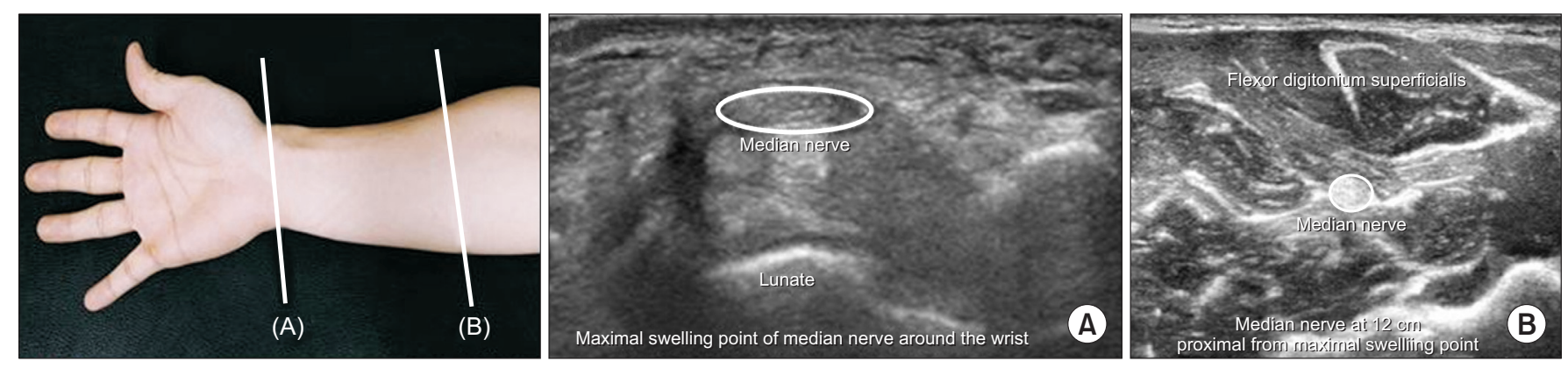

Fig. 1. Ultrasonographic probe position at two different levels. Ultrasonographic transverse scanning was done. The cross-sectional areas of the maximal swelling point of the median nerve were measured at the wrist (A) and $12 \mathrm{~cm}$ proximal to this level (B). 
Table 2. Baseline characteristics of the study participants

\begin{tabular}{lccccc}
\hline & $\begin{array}{c}\text { Group 1 } \\
\text { (non-DM+non-CTS) }\end{array}$ & $\begin{array}{c}\text { Group 2 } \\
\text { (non-DM+CTS) }\end{array}$ & $\begin{array}{c}\text { Group 3 } \\
\text { (DM+non-CTS) }\end{array}$ & $\begin{array}{c}\text { Group 4 } \\
\text { (DM+CTS) }\end{array}$ & p-value \\
\hline No. of subjects & 77 & 162 & 20 & 94 & \\
Age (yr) & $59.9 \pm 11.3$ & $52.2 \pm 8.1$ & $66.2 \pm 8.3$ & $59.1 \pm 10.4$ & $<0.001^{*}$ \\
Female & $46(59.7)$ & $147(90.7)$ & $9(45.0)$ & $83(88.3)$ & $<0.001^{*}$ \\
Height $(\mathrm{cm})$ & $161.2 \pm 9.5$ & $155.2 \pm 5.7$ & $159.6 \pm 8.2$ & $153.8 \pm 6.6$ & $<0.001^{*}$ \\
Weight $(\mathrm{kg})$ & $60.2 \pm 9.9$ & $59.0 \pm 8.2$ & $60.1 \pm 8.3$ & $60.1 \pm 8.1$ & 0.811 \\
BMI $\left(\mathrm{kg} / \mathrm{m}^{2}\right.$ ) & $23.1 \pm 3.1$ & $24.5 \pm 2.9$ & $23.5 \pm 3.1$ & $25.4 \pm 2.5$ & $<0.001^{*}$ \\
\hline DM duration $(\mathrm{yr})$ & NA & NA & $5.2 \pm 4.6$ & $4.7 \pm 2.1$ & \\
\hline
\end{tabular}

Values are presented a mean \pm standard deviation or number (\%).

DM, diabetes mellitus; CTS, carpal tunnel syndrome; BMI, body mass index; NA, not available.

${ }^{*} \mathrm{p}<0.05$, using the ANOVA.

Table 3. Parameters of ultrasonography among the groups

\begin{tabular}{lccccc} 
& $\begin{array}{c}\text { Group 1 } \\
\text { (non-DM+non-CTS) }\end{array}$ & $\begin{array}{c}\text { Group 2 } \\
(\text { non-DM+CTS) }\end{array}$ & $\begin{array}{c}\text { Group 3 } \\
(\text { DM+non-CTS) }\end{array}$ & $\begin{array}{c}\text { Group 4 } \\
(\text { DM+CTS) }\end{array}$ & p-value \\
\hline CSA $\left(\mathrm{mm}^{2}\right)$ & $8.5 \pm 0.5^{\mathrm{a}, \mathrm{b}, \mathrm{c})}$ & $14.7 \pm 0.4^{\mathrm{a})}$ & $11.8 \pm 1.0^{\mathrm{b}, \mathrm{e})}$ & $15.9 \pm 0.5^{\mathrm{c}, \mathrm{e})}$ & $<0.001$ \\
WFR & $1.1 \pm 0.1^{\mathrm{a}, \mathrm{c})}$ & $2.5 \pm 0.1^{\mathrm{a}, \mathrm{d})}$ & $1.4 \pm 0.3^{\mathrm{d}, \mathrm{e})}$ & $2.9 \pm 1.4^{\mathrm{c}, \mathrm{e})}$ & $<0.001$ \\
\hline
\end{tabular}

Values are presented as mean \pm standard deviation.

DM, diabetes mellitus; CTS, carpal tunnel syndrome; CSA, cross-sectional area; WFR, wrist to forearm ratio.

Adjusted for baseline value of age and body mass index. A p-value was calculated by ANCOVA among groups. According to post hoc analyses, ${ }^{\text {a) }} \mathrm{p}<0.001$, groups 1 vs. $2,{ }^{\text {b) }} \mathrm{p}<0.001$, groups $1 \mathrm{vs.} 3,{ }^{\mathrm{c})} \mathrm{p}<0.001$, groups $1 \mathrm{vs.} 4,{ }^{\mathrm{d})} \mathrm{p}<0.001$, groups 2 vs. 3 , and ${ }^{\mathrm{e})} \mathrm{p}<0.001$, groups 3 vs. 4 .

In a post-hoc comparison, CSA and WFR values were significantly greater in CTS patients regardless of DM compared to non-CTS patients $(\mathrm{p}<0.01)$. In non-CTS patients, the CSA and WFR values were significantly greater compared to non-DM patients $(\mathrm{p}<0.01)$.

\section{The Cut-off value CSA and WFR for diagnosis of CTS}

The ROC curve analysis revealed a CSA $\geq 10.1 \mathrm{~mm}^{2}$ (area under curve $[\mathrm{AUC}]=0.89$, sensitivity $=84.8 \%$, and specificity $=88.7 \%$ ) and a WFR $\geq 1.55$ (AUC=0.95, sensitivity $=91.4 \%$, and specificity $=92.8 \%$ ) as the most powerful diagnostic values for CTS in patients with or without DM (Fig. 2A). The ROC curve analysis of DM patients (Fig. 2B) revealed a $C S A \geq 12.5 \mathrm{~mm}^{2}$ (AUC $=0.77$, sensitivity $=78.7 \%$, and specificity $=65.0 \%$ ) and a WFR $\geq 1.87$ (AUC $=0.88$, sensitivity $=80.9 \%$, and specificity $=80.0 \%$ ) as the most powerful diagnostic values for CTS. The ROC curve analysis of non-DM patients (Fig. 2C) revealed a CSA $\geq 10.0 \mathrm{~mm}^{2}$ (AUC $=0.93$, sensitivity $=88.3 \%$, and specificity $=88.3 \%$ ) and a WFR $\geq 1.52$ (AUC $=0.97$, sensitivity $=90.7 \%$, and specificity $=89.6 \%$ ) as the most powerful diagnostic values for CTS.

\section{DISCUSSION}

This study has several major findings. First, the CSA and WFR were larger in DM patients (excluding polyneuropathy) compared to non-DM patients in both non-CTS and CTS hands. Second, the diagnostic cut-off values of the CSA and WFR differed according to the presence or absence of DM. Lastly, based on the ROC analysis, WFR was observed to have a greater diagnostic value than the CSA of the median nerve at the maximal swelling site alone, irrespective of the DM status.

Numerous studies have shown the utility of ultrasound in the diagnosis of CTS $[9,10]$. However, only a few studies have reported the importance of ultrasonographic measurements in discrimination between non-DM and DM patients with CTS, further confirmed by electrodiagnosis (excluding polyneuropathy).

Many studies have explained the enlargement of the median nerve in CTS employing a histopathological approach. In terms of the biologic response to compression of the peripheral nerve, it has been reported that the 

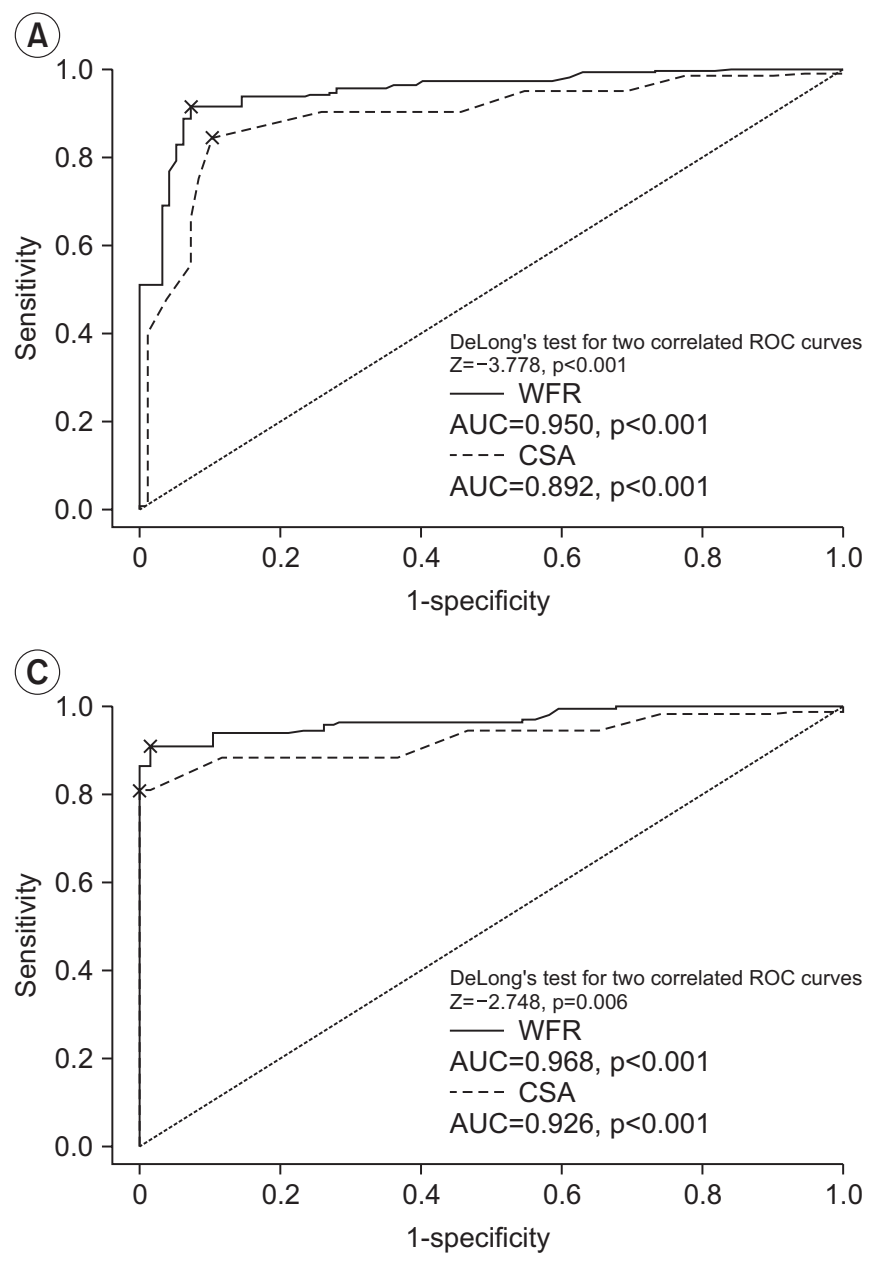

destruction of the blood-nerve barrier ischemia induces a cascade comprising of endoneurial edema, demyelination, perineural inflammation, axonal degeneration, generation of new axons, re-myelination, fibrosis, and thickening of the perineurium and endothelium $[11,12]$. Moreover, diabetes mellitus influences the susceptibility of the median nerve via the oxidative stress, (which initiates and amplifies neuro-inflammation) and/or the polyol pathway, (which includes glycation and proinflammatory reactions) $[13,14]$. Previous reports showed no significant difference in the wrist CSA of DM patients with axonal (including diabetic) polyneuropathy compared to that of the healthy volunteers $[6,7]$. However, Pitarokoili et al. [15] described the ultrasonographic changes in the peripheral nerves in diabetes and reported enlarged median nerve CSA at compression sites irrespective of the presence of peripheral polyneuropathy. Our results revealed larger CSA and WFR values in patients with DM than in non-DM patients. The differ-

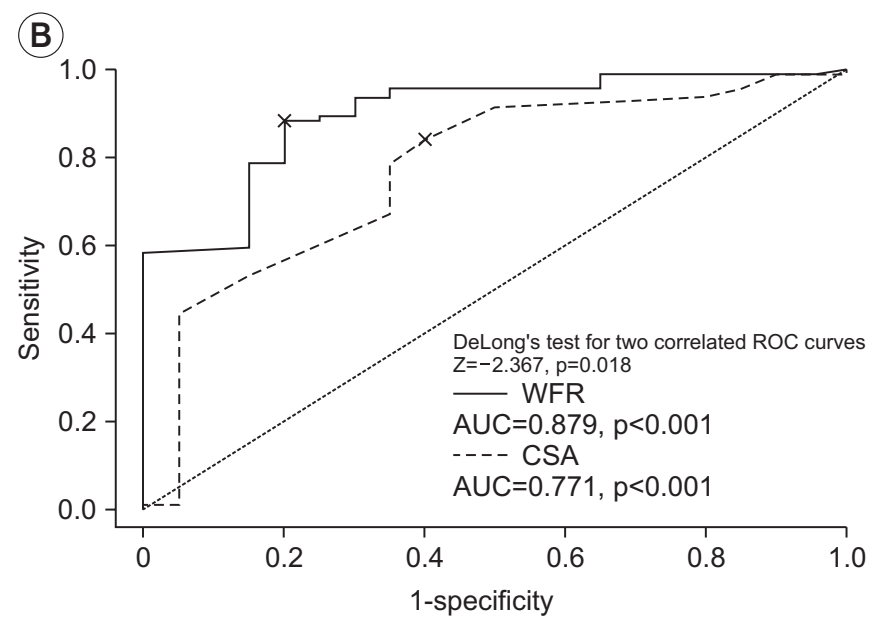

Fig. 2. The receiver operating characteristic (ROC) curve analysis of the CSA and WFR values for the diagnosis of carpal tunnel syndrome in total patients (A), DM patients (B), and non-DM patients (C). CSA, cross-sectional area; WFR, wrist to forearm ratio; AUC, area under the curve; $\mathrm{DM}$, diabetes mellitus.

ences in CSA and WFR between the two groups might be attributed to the increased vulnerability of the nerves of DM patients to external compression. The observed differences in the EDX parameters of CTS patients according to DM status corroborate that association.

Many reports have proposed a range of cut-off values for the CSA of the median nerve, and the thresholds reported for the diagnosis of CTS vary from 9 to $15 \mathrm{~mm}^{2}$ [16]. However, the currently established diagnostic cut-off values using ultrasonography were not tailored for patients with DM, which is considered an independent risk factor for CTS. We suggest the application of different cut-off values for patients with and without DM. Furthermore, the present study excluded DM patients with polyneuropathy, which was confirmed based on nerve conduction studies and medical record reviews in order to focus on the impact of DM alone. We determined the median nerve CSA cut-off value to be more than $10 \mathrm{~mm}^{2}$ (sensitivity $=88.3 \%$, specificity $=88.3 \%$ ) for non-DM patients and 
$12.5 \mathrm{~mm}^{2}$ (sensitivity $=80.9 \%$, specificity $=80.0 \%$ ) for DM patients.

The measurement of median CSA is accepted as the reliable diagnostic method but the CSA alone measurement may vary depending on the researchers and correlates with other patient's characteristics factors. Therefore, WFR has been suggested as a more accurate diagnostic index for CTS [9]. In this study, CSA values were found to be higher in diagnosis either CTS or DM, though the diagnostic value of the CSA was not significantly different between groups 2 and 3. However, significant differences were observed in the diagnostic value of the WFR between groups 2 and 3. Consequently, the WFR was more highly associated with the diagnosis of CTS than the CSA. Moreover, the AUC value of the WFR obtained by ROC analysis was higher, especially in DM patients.

This study has some limitations. First, it was a relatively small-scale and single center-based study and the number of patients in group 3 was particularly small since the majority of DM patients visiting the outpatient clinic have clinical symptoms relevant to diabetic polyneuropathy and comes for confirmation of polyneuropathy. Second, ultrasonography is an operator-dependent test. The use of a single operator and the absence of repeated measurements are the weaknesses of this study. Third, it was not possible to exclude the association with factors related to symptoms, (e.g., the duration or, severity) of the DM or CTS. Therefore, future large-scale prospective studies are needed to confirm the role of DM in CTS pathophysiology.

In conclusion, DM patients showed increased CSA and WFR values compared to patients without DM. In the use of ultrasonographic assessment for the diagnosis of CTS, distinct cut-off values for patients with DM are necessary. Lastly, the ROC analysis revealed that a more precise diagnosis of CTS could be possible if WFR can be applied in place of CSA.

\section{CONFLICT OF INTEREST}

No potential conflict of interest relevant to this article was reported.

\section{REFERENCES}

1. Werner RA, Andary M. Carpal tunnel syndrome: pathophysiology and clinical neurophysiology. Clin Neurophysiol 2002;113:1373-81.

2. Wong SM, Griffith JF, Hui AC, Lo SK, Fu M, Wong KS. Carpal tunnel syndrome: diagnostic usefulness of sonography. Radiology 2004;232:93-9.

3. Jeong JS, Yoon JS, Kim SJ, Park BK, Won SJ, Cho JM, et al. Usefulness of ultrasonography to predict response to injection therapy in carpal tunnel syndrome. Ann Rehabil Med 2011;35:388-94.

4. Pourmemari MH, Shiri R. Diabetes as a risk factor for carpal tunnel syndrome: a systematic review and meta-analysis. Diabet Med 2016;33:10-6.

5. Breiner A, Qrimli M, Ebadi H, Alabdali M, Lovblom LE, Abraham A, et al. Peripheral nerve high-resolution ultrasound in diabetes. Muscle Nerve 2017;55:171-8.

6. Moon HI, Kwon HK, Kim L, Lee HJ, Lee HJ. Ultrasonography of palm to elbow segment of median nerve in different degrees of diabetic polyneuropathy. Clin Neurophysiol 2014;125:844-8.

7. Zaidman CM, Al-Lozi M, Pestronk A. Peripheral nerve size in normals and patients with polyneuropathy: an ultrasound study. Muscle Nerve 2009;40:960-6.

8. American Diabetes Association. Diagnosis and classification of diabetes mellitus. Diabetes Care 2011;34 Suppl 1:S62-9.

9. Kang S, Kwon HK, Kim KH, Yun HS. Ultrasonography of median nerve and electrophysiologic severity in carpal tunnel syndrome. Ann Rehabil Med 2012;36:729.

10. Kotevoglu N, Gülbahce-Saglam S. Ultrasound imaging in the diagnosis of carpal tunnel syndrome and its relevance to clinical evaluation. Joint Bone Spine 2005;72:142-5.

11. Rempel D, Dahlin L, Lundborg G. Pathophysiology of nerve compression syndromes: response of peripheral nerves to loading. J Bone Joint Surg Am 1999;81:160010.

12. Yayama T, Kobayashi S, Nakanishi Y, Uchida K, Kokubo Y, Miyazaki T, et al. Effects of graded mechanical compression of rabbit sciatic nerve on nerve blood flow and electrophysiological properties. J Clin Neurosci 2010;17:501-5.

13. Sandireddy R, Yerra VG, Areti A, Komirishetty P, Kumar A. Neuroinflammation and oxidative stress in diabetic neuropathy: futuristic strategies based on these targets. Int J Endocrinol 2014;2014:674987. 
14. Yagihashi S. Recent advances in clinical practice and in basic research on diabetic neuropathy. Brain Nerve 2011;63:571-82.

15. Pitarokoili K, Kerasnoudis A, Behrendt V, Labedi A, Ayzenberg I, Gold R, et al. Facing the diagnostic challenge: Nerve ultrasound in diabetic patients with neu- ropathic symptoms. Muscle Nerve 2016;54:18-24.

16. Tajika T, Kobayashi T, Yamamoto A, Kaneko T, Takagishi K. Diagnostic utility of sonography and correlation between sonographic and clinical findings in patients with carpal tunnel syndrome. J Ultrasound Med 2013; 32:1987-93. 